\title{
Identification of a key candidate gene-phenotype network mediated by glycyrrhizic acid using pharmacogenomic analysis
}

\author{
SHIQUN WANG ${ }^{1}, \mathrm{LU} \mathrm{LI}^{2}$ and LONG SHI ${ }^{1,3}$ \\ ${ }^{1}$ Xiaoshan Biotechnology Center, Yangtze Delta Region Institute of Tsinghua University, Hangzhou, \\ Zhejiang 311231; ${ }^{2}$ Department of Nephrology, Affiliated Children's Hospital of Zhejiang University, \\ Hangzhou, Zhejiang 310052; ' Zhejiang Provincial Key Laboratory of Applied Enzymology, \\ Yangtze Delta Region Institute of Tsinghua University, Jiaxing, Zhejiang 314006, P.R. China
}

Received January 7, 2019; Accepted June 27, 2019

DOI: $10.3892 / \mathrm{mmr} .2019 .10494$

\begin{abstract}
Glycyrrhizic acid (GA) is primarily used as an anti-inflammatory agent in cases of chronic hepatitis. However, its underlying mechanisms in diverse biological processes and its reported benefits are yet to be fully elucidated. In the current study, an analytical method based on pharmacogenomics was established to mine disease-modulatory activities mediated by GA. Five primary protein targets and 138 functional partners were identified for GA by querying open-source databases, including Drugbank and STRING. Subsequently, GA-associated primary and secondary protein targets were integrated into Cytoscape to construct a protein-protein interaction network to establish connectivity. GA-associated target genes were then clustered based on Gene Ontology and Kyoto Encyclopedia of Genes and Genomes pathway enrichment analysis. The tumor necrosis factor axis was revealed to be a primary module regulated by GA-associated targets. Furthermore, 12 hub genes were queried to assess their potential anti-cancer effects using cBioPortal. The results indicated that pharmacogenomics-based analysis improved understanding of the underlying drug-target events of GA and provided predictive and definitive leads for future studies.
\end{abstract}

\section{Introduction}

Glycyrrhizic acid (GA) is a triterpene glycoside isolated from the root of the licorice plant. In Japan and China, GA has been developed as an anti-inflammatory hepatoprotective drug in cases of chronic hepatitis (1). GA has also been determined

Correspondence to: Mr. Shiqun Wang, Xiaoshan Biotechnology Center, Yangtze Delta Region Institute of Tsinghua University, 495 Hongken Road, Xiaoshan Innovation Polis, Hangzhou, Zhejiang 311231, P.R. China

E-mail: wangshiqun@tsinghua-zj.edu.cn

Key words: glycyrrhizic acid, pharmacogenomic analysis, cancer, targets, tumor necrosis factor axis to exhibit a wide range of pharmacological and biological activities, including anti-ulcer, anti-allergic, antioxidant, anti-diabetic and anti-tumor effects (2). In recent years, an increasing number of studies have focused on determining the underlying mechanisms and targets of GA (3-14). However, the lack of systematic dissection of drug-target mechanisms between GA-associated primary and secondary targets is a great obstacle to the research and development of susceptibility indicators for clinical applications. To address this obstacle, the following should be determined: i) Whether significant interactions exist between recently reported targets, including high mobility group box 1 (HMGB1), and well-documented targets mediated by GA, including caspase-3 (CASP3), tumor necrosis factor (TNF) and nuclear factor $\kappa \mathrm{B}$ subunit 2 (NFKB2) $(1,8,14)$; ii) whether there are key hub elements among targets that can improve the understanding of interrelated biological actions mediated by GA, including inflammatory tumors or apoptosis regulation in immune-associated diseases; and iii) whether the systematic assessment of previously published articles can provide predictive leads to uncover molecular events mediated by GA.

In recent years, the genetic alteration of $\sim 20$ genes has impacted $~ 80$ approved drugs and, as such, has affected their applicability in a clinical setting (15). Pharmacogenomics (PG) has been developed to determine the associations between genetic variants and drug responses and to create an evidenced-based strategy for improving the administration of drugs (16). To remove analytical obstacles, the PG-based approach may be suitable for mining existing drug-target data obtained from numerous multicenter genomics studies. PG may also be effective for focusing research scope and direction as well as determining drug-target interactions. The current study queried multiple online sources (Drugbank, PubChem, PharmaGKB, STRING, DAVID and cBioPortal) to optimize candidate target genes and to construct a functional activity network associated with GA. The results revealed that the main target pathway mediated by GA is closely associated with the TNF axis. Furthermore, an overlapping gene set extracted from protein-protein interaction (PPI) and Kyoto Encyclopedia of Genes and Genomes (KEGG) pathway analyses may be the key element to the pathogenesis of neuroendocrine prostate cancer, which is a rare malignancy with a poor prognosis, 
associated with inflammatory induction, and mostly occurs as an adaptive response following intensive androgen deprivation treatment (17). Thus, the present study provided an insight into GA-mediated anti-cancer activity and may have implications for the treatment of inflammatory malignant neuroendocrine prostate cancer.

\section{Materials and methods}

Identification of drug targets and visualization of interactions. GA drug-target information was searched using multiple online sources, including Drugbank (https://www. drugbank.ca/), PharmaGKB (https://www.pharmgkb.org/) and PubChem (https://pubchem.ncbi.nlm.nih.gov/). Identification of the direct protein targets of GA was performed using DrugBank. A total of five primary protein targets associated with GA were identified. Interaction networks for GA-associated primary protein targets were generated from the STRING database (https://string-db. org/) and integrated into seven active interaction sources (textmining, experiments, databases, co-expression, neighborhood, gene fusion and co-occurrence). The data were then used to output a total of 138 functional partners. The visualized interaction network of integrated GA and GA target data was produced using Cytoscape (version 3.61; https://cytoscape.org/), an open-source software package for visualizing complex networks. Bioinformatics analysis was performed as previously described (18).

Gene Ontology $(G O)$ and KEGG analysis. Functional and pathway enrichment for the target gene set of GA were analyzed using DAVID (version 6.8; https://david.ncifcrf. gov/), a tool for gene functional annotation, visualization and integrated discovery (19). A total of 129 GA-associated target genes were specifically queried and integrated into the GO and KEGG analysis tools in DAVID. The top $30 \mathrm{GO}$ terms with $\mathrm{P}<0.05$ are presented. The significance of the identified genes from the drug-target analysis was verified by replacing significant candidates with other random genes. Validated genes were then systematically sorted based on KEGG analysis. The top 10 pathways with $\mathrm{P}<0.05$ were selected. Statistical analysis was subsequently performed using DAVID, and data were analyzed using the Fisher's exact test.

Analysis of GA-associated tumor genetic alterations using cBioPortal. To determine the connectivity of GA-associated target genes within different cancer genomic studies, GA-associated data were integrated for further analysis using cBioPortal (http://www.cbioportal.org/), an open-source platform that provides visualization, analysis and downloadable large-scale cancer genomic datasets (20). The visualization of genetic alterations across tumor samples was produced using OncoPrint tool. Interaction networks of the overlapping genes from the enrichment analysis were generated via multiple visualization analyses. The underlying data could then be connected to clinical profiles to promote novel discoveries in drug-associated biological functions. The overlapping genes of pathway enrichment analysis was performed with Venn analysis (Venny software; version 2.1; http://bioinfogp.cnb. csic.es/tools/venny/index.html).

\section{Results}

Characterization of $G A$ bioactivity and visualization of the GA-associated target network via Cytoscape. Drugbank and PubChem are free bioinformatics and cheminformatics resources that allow for the mining of detailed drug data with comprehensive drug-target information (21). The current study queried Drugbank using GA as the input and subsequently checked against PubChem. This resulted in output DB13751, categorizing GA as an anti-inflammatory agent used in the alimentary tract and for liver therapy, grouping GA as an approved experimental drug. Five primary protein targets of GA [corticosteroid 11- $\beta$-dehydrogenase isozyme 1 (HSD11B1), TNF, CASP3, NFKB2 and lipoprotein lipase (LPL)] were revealed (Table I). To further expand the analyses, the secondary protein targets of GA were detected via PPI analysis using STRING. A total of 138 functional partners were identified and determined to be associated with GA-associated primary protein targets (Table SI).

A drug-target interaction network was constructed by integrating data into Cytoscape (version 3.61) (18). The primary and secondary PPIs of GA, including HSD11B1 (5 PPIs), TNF (44 PPIs), CASP3 (23 PPIs), NFKB2 (38 PPIs) and LPL (28 PPIs), are presented in Fig. 1. Additionally, when compared with HSD11B1 and LPL, three primary protein targets (TNF, NFKB2 and CASP3) possessed up to 10 crossed secondary protein targets [baculoviral IAP repeat-containing protein 3 (BIRC3), BIRC2, FAS-associated death domain protein (FADD), 26S proteasome non-ATPase regulatory subunit 2 (PSMD2), NF- $\kappa \mathrm{B}$ essential modulator (IKBKG), ubiquitin C (UBC), receptor-interacting serine/threonine-protein kinase 1 (RIPK1), NF- $\kappa$ B subunit 1 (NFKB1), $26 \mathrm{~S}$ proteasome regulatory subunit $6 \mathrm{~A}$ (PSMC3) and interleukin $1 \beta$ (IL1B)]. This indicated that the main target pathway mediated by GA is closely associated with the TNF axis.

GO analysis and signaling pathway enrichments connected to GA-associated gene sets. To assess the functional attributes of a GA-associated gene set (129 genes from Homo sapiens), GO and pathway enrichment analyses were performed using DAVID with $\mathrm{P}<0.05$ as the cut-off criterion (19). As presented in Fig. 2, GA-associated genes were classified into three functional groups: Biological processes (BP), cellular components $(\mathrm{CC})$ and molecular functions $(\mathrm{MF})$. In the BP group, genes were mainly enriched in 'TNF-mediated signaling pathway' and 'NIK/NF- $\kappa \mathrm{B}$ signaling'. In the $\mathrm{CC}$ group, genes were largely enriched in 'cytosol', 'proteasome complex' and 'nucleoplasm'. In the MF group, genes were primarily enriched in 'protein binding'. The GO results therefore indicated that most GA-mediated genes were significantly enriched in 'TNF-mediated signaling pathway', 'protein binding', 'cytosol' and 'nucleoplasm'.

The top 10 KEGG pathways connected to GA-associated genes are presented in Table II and were as follows: 'TNF signaling pathway' (34 genes), 'Epstein-Barr virus infection' (41 genes), 'NF-кB signaling pathway' (28 genes), 'apoptosis' (24 genes), 'proteasome' (20 genes), 'herpes simplex infection' (28 genes), 'NOD-like receptor signaling pathway' (18 genes), 'toxoplasmosis' (23 genes), 'osteoclast differentiation' (23 genes) and 'measles' (22 genes). In this 
Table I. Identification of primary protein targets of glycyrrhizic acid using DrugBank.

\begin{tabular}{|c|c|c|c|c|c|}
\hline \multicolumn{2}{|c|}{ Searched drug } & \multicolumn{4}{|c|}{ Targets } \\
\hline \# & DB_ID & Gene_Symbol & Uniprot_ID & Entrez_ID & Actions \\
\hline 1 & DB13751 & HSD11B1 & P28845 & 3290 & Antagonist \\
\hline 2 & DB13751 & TNF & P01375 & 7124 & Antagonist \\
\hline 3 & DB13751 & CASP3 & P42574 & 836 & Antagonist \\
\hline 4 & DB13751 & NFKB2 & Q00653 & 4791 & Translocation inhibitor \\
\hline 5 & DB13751 & LPL & P06858 & 4023 & Inducer \\
\hline
\end{tabular}

HSD11B1, corticosteroid 11- $\beta$-dehydrogenase isozyme 1; TNF, tumor necrosis factor; CASP3, caspase-3; NFKB2, nuclear factor $\kappa B$ subunit 2; LPL, lipoprotein lipase.

Table II. List of significant enriched signaling pathways of the glycyrrhizic acid-associated genes.

\begin{tabular}{|c|c|c|c|}
\hline Pathway name & \#Gene & P-value & Entrez_ID \\
\hline TNF signaling pathway & 34 & $1.97 \times 10^{-33}$ & $\begin{array}{l}718514377186634771244792479068852077132953023538367133 \\
602355311477187338335695970877233010454231183298717843 \\
3725143287378517110357128\end{array}$ \\
\hline Epstein-Barr virus infection & 41 & $2.99 \times 10^{-33}$ & $\begin{array}{l}718571864793743179794792479047916885207570734585708 \\
57095710986157131147571471873383597059717157592510454 \\
23118871772975705570457185702571737258737143257015700 \\
85177128\end{array}$ \\
\hline $\mathrm{NF}-\kappa \mathrm{B}$ signaling pathway & 28 & $2.78 \times 10^{-27}$ & $\begin{array}{l}718571867124331479247904791688571323553114771873383 \\
405559705971115650330104542311832987175588106739598737 \\
85177128\end{array}$ \\
\hline Apoptosis & 24 & $2.24 \times 10^{-25}$ & $\begin{array}{l}71867124331597054205715747924790877233032987178797207 \\
843713283983687438795581873785171147\end{array}$ \\
\hline Proteasome & 20 & $1.31 \times 10^{-22}$ & $\begin{array}{l}5699797957055718568957045717568657025701568434585707 \\
5700570857095710986157135714\end{array}$ \\
\hline Herpes simplex infection & 28 & $4.51 \times 10^{-18}$ & $\begin{array}{l}718571867124634747934792479068857132235383634583553 \\
845411477187356959705420571578772650010454231187297 \\
372585173593\end{array}$ \\
\hline $\begin{array}{l}\text { NOD-like receptor signaling } \\
\text { pathway }\end{array}$ & 18 & $2.19 \times 10^{-17}$ & $\begin{array}{l}3569712463475970360647934792479033010454 \\
32923118688514328517355371281147\end{array}$ \\
\hline Toxoplasmosis & 23 & $4.57 \times 10^{-17}$ & $\begin{array}{l}71243314793597054205794444792479033010454329231186885 \\
2077297713283695914323458851735931147\end{array}$ \\
\hline Osteoclast differentiation & 23 & $4.67 \times 10^{-16}$ & $\begin{array}{l}71867124597054685971479247904791104542311868857297207 \\
7132154023533725143285173458355311473552\end{array}$ \\
\hline Measles & 22 & $9.04 \times 10^{-15}$ & $\begin{array}{l}3565356947935970715747924790231186885879720772975588 \\
874387953458355335937128355211473558\end{array}$ \\
\hline
\end{tabular}

TNF, tumor necrosis factor.

functional enrichment analysis, the GA-associated gene set was indicated to be closely associated with TNF and TNF-related signaling pathways, which possess potential biological functions, including: i) The regulation of gene transcription associated with survival, proliferation and inflammation via the $\mathrm{TNF} / \mathrm{NF}-\kappa \mathrm{B}$ signaling pathway; and ii) the control of cell apoptosis via the TNF/caspase family signaling pathway. On account of a direct association with the TNF axis, as determined via the interactive network analysis of GA-associated protein targets, the current study focused the subsequent analysis on the TNF and TNF-associated signaling pathways (Fig. 1). Thus, based on the results of the GO and KEGG pathway analysis, in combination with the determination of GA-associated protein targets, it was revealed that the 


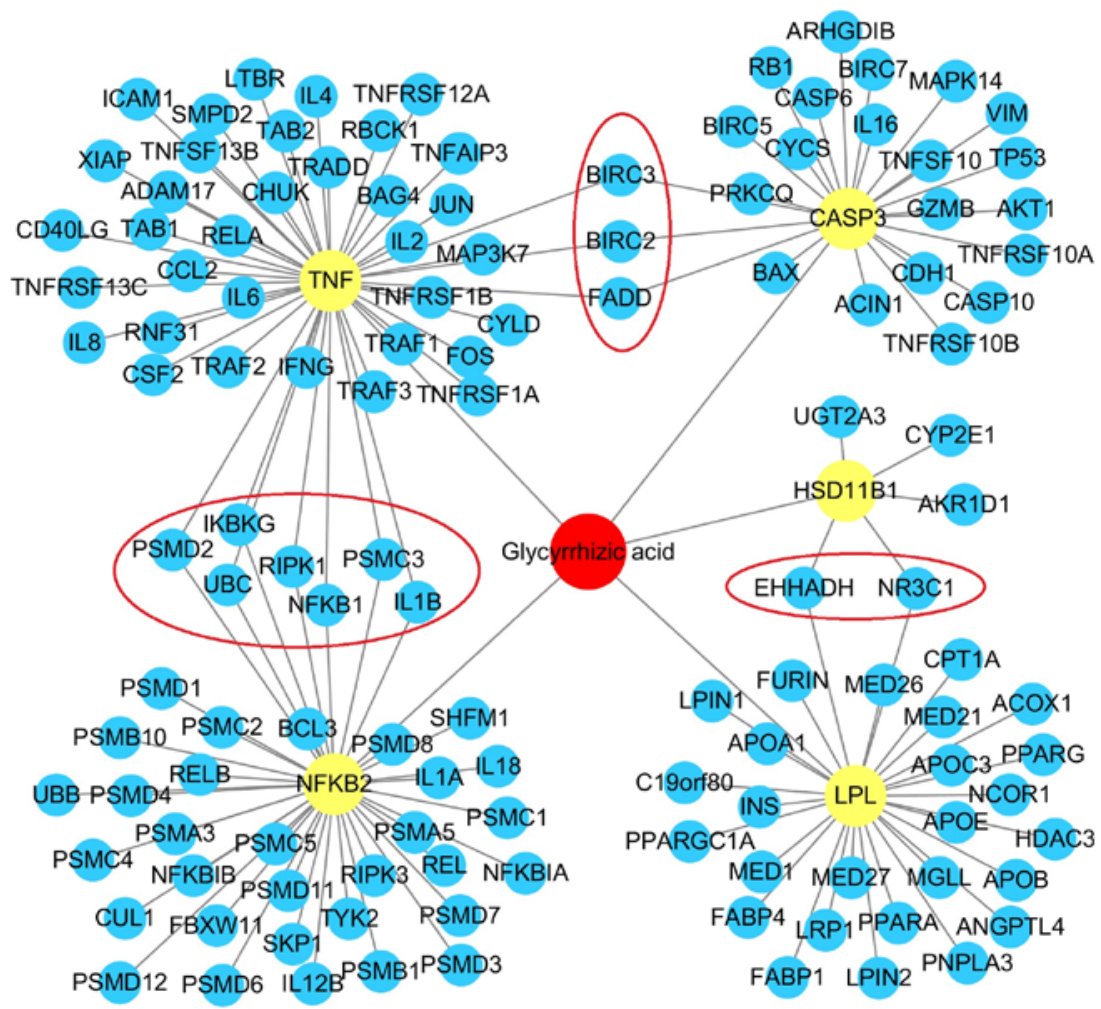

Figure 1. Drug-target interaction network of GA-associated protein targets. GA is presented in red, primary protein targets including HSD11B1, TNF, CASP3, NFKB2 and LPL are presented in yellow, and secondary protein targets that interact with GA-associated primary protein targets are presented in blue. Crossed secondary protein targets (BIRC3, BIRC3, FADD, PSMD2, IKBKG, UBC, RIPK1, NFKB1, PSMC3, IL1B, EHHADH are NR3C1) are presented as red circles. The network exhibited a total of 138 protein-protein interactions. GA, glycyrrhizic acid.

\section{Significant enriched GO Terms (Top 30)}

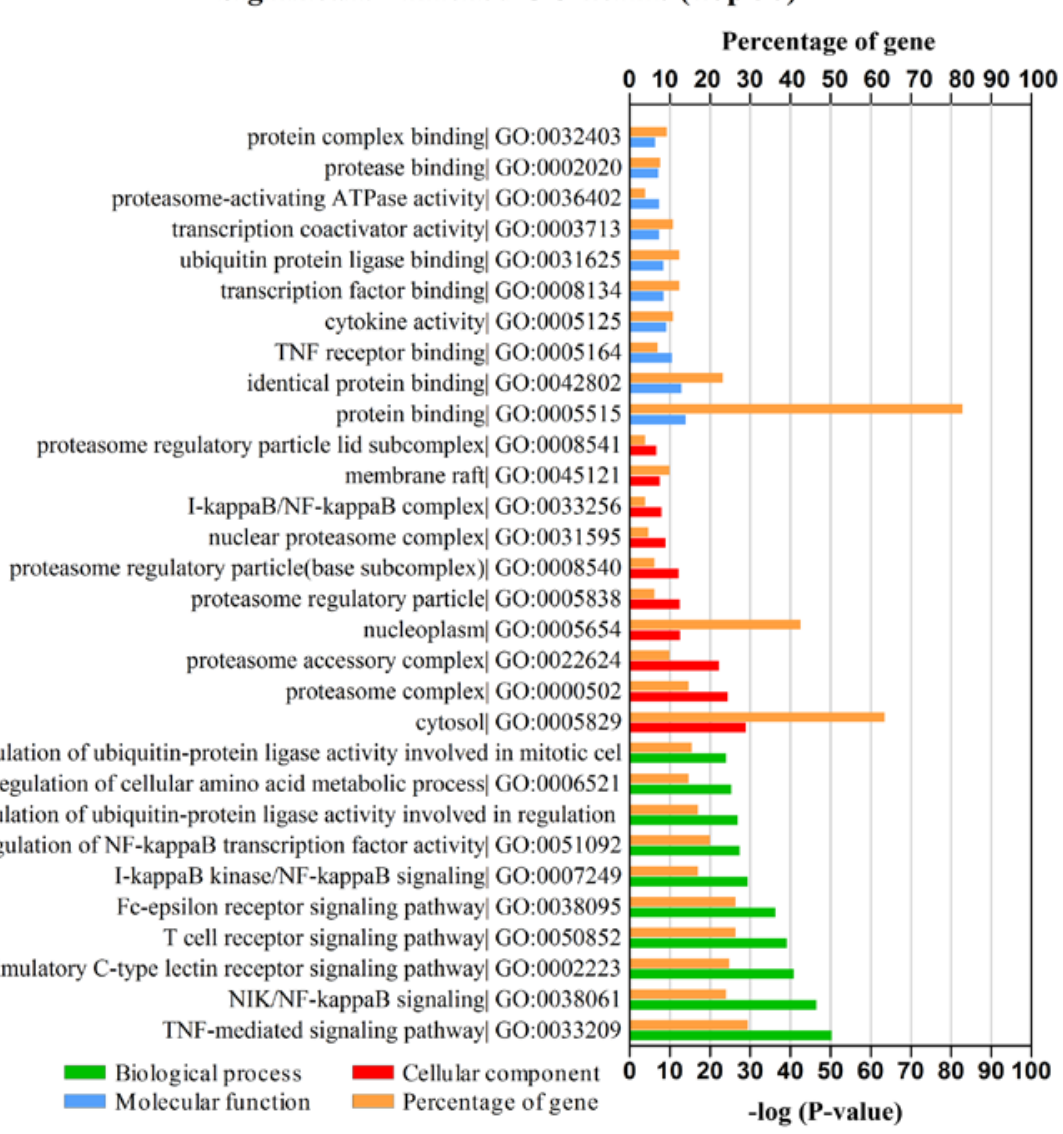

Figure 2. GO analysis and significant enriched GO terms of glycyrrhizic acid-associated genes. GO, Gene Ontology. 
A Summary of alterations for 12 overlapping genes in cBioPortal studies (top 10)

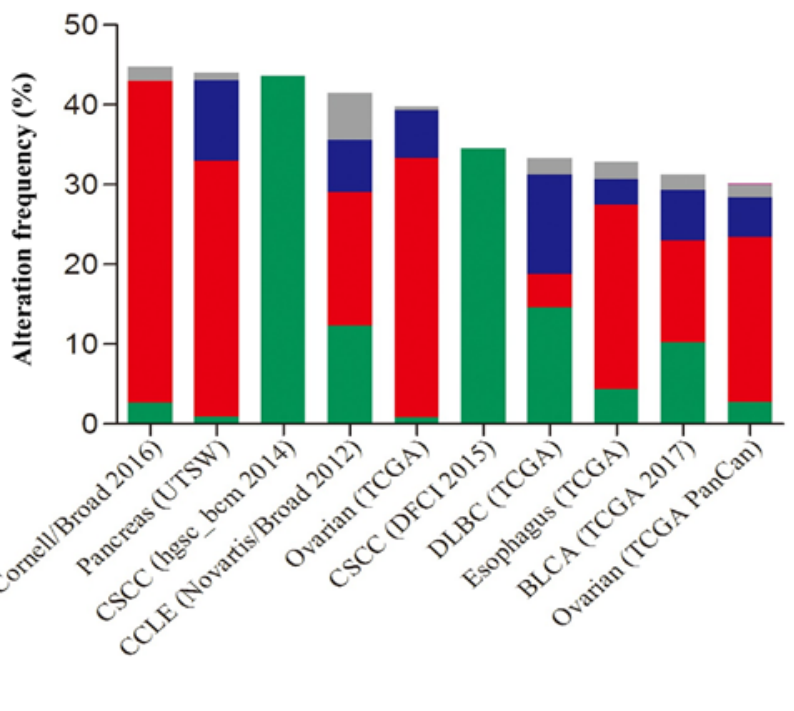

B

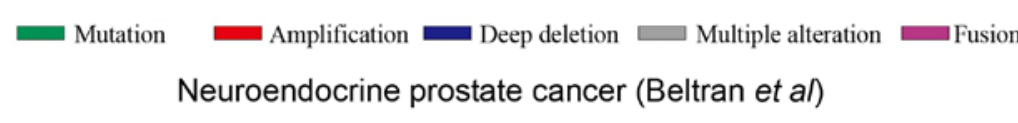

Tumor samples with sequencing and CNA data (107 samples )/ 12 genes

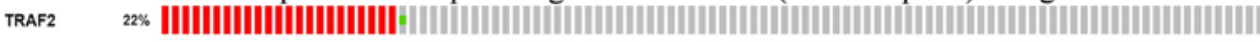

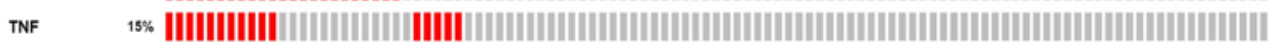

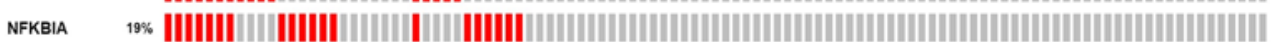

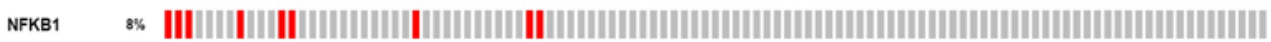

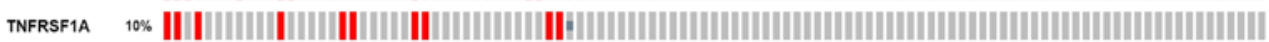

сн4к $11 \%$ I/I

réa $\quad 26 \%$ |

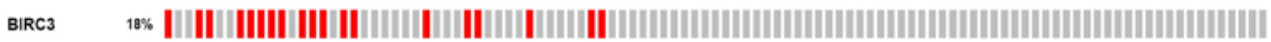

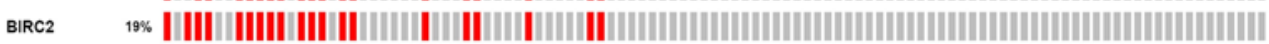

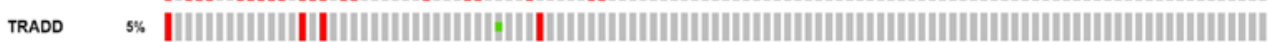

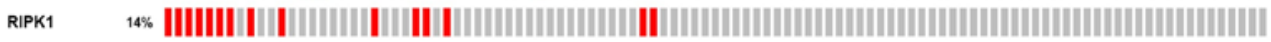

Іквк $\quad$ 26\%

Genetic Alteration || Missense Mutation (unknown significance) i Truncating Mutation (unknown signifcance) | Amplifcation | No alterations

Case Set: Tumor Samples with sequencing and CNA data (77 patients / 107 samples)

Figure 3. Summary of the genetic changes of 12 GA-associated overlapping genes, obtained from cBioPortal cancer genomic studies. (A) Overview of the top 10 genomic changes of 12 overlapping genes (TRAF2, TNF, NFKBIA, NFKB1, TNFRSF1A, CHUK, RELA, BIRC3, BIRC2, TRADD, RIPK1 and IKBKG) in cBioPortal genomic datasets available from all 225 listed studies. (B) OncoPrint profile of the genetic alterations of 12 overlapping genes in samples of neuroendocrine prostate cancer (Beltran et al) (24). The results revealed alterations in 51 (48\%) of 107 sequenced samples. CNA, copy-number alteration; GA, glycyrrhizic acid.

main biological and functional association exists between $\mathrm{GA}$ and the key hub elements of TNF and the TNF-associated pathway (crossed targets including BIRC2, BIRC3, RIPK1, IKBKG and NFKB1; Fig. 1), which are closely associated with certain physiological or pathological regulatory mechanisms including apoptosis, anti-apoptosis, cell survival, cell proliferation, cell differentiation and inflammation (22).

Mining genetic alterations of GA-associated genes in cBioPortal cancer studies. Although DrugBank categorizes GA as an anti-inflammatory agent used for hepatic protection or peptic ulcer treatment, the results of the enrichment analysis indicated that it may also be associated with apoptosis, survival and proliferation. Furthermore, a previous study revealed that GA demonstrates marked efficacy in the treatment of inflammation-induced migration or invasion in pancreatic cancer (23). To further confirm if susceptible cancers are associated with GA-associated genes, cBioPortal was utilized to analyze the genetic alterations of GA-associated genes in different types of human cancer. Since the TNF axis is a main target module of GA (Fig. 1), and since the NF- $\mathrm{B}$ and apoptosis pathways are associated with the TNF signaling pathway, an overlapping gene set was extracted [TNF receptor associated factor 2 (TRAF2), TNF, NFKB inhibitor- $\alpha$ (NFKBIA), NFKB1, TNF receptor superfamily member $1 \mathrm{~A}$ (TNFRSF1A), component of inhibitor of nuclear factor- $\kappa \mathrm{B}$ kinase complex (CHUK), RELA proto-oncogene, NF- $\kappa \mathrm{B}$ subunit (RELA), BIRC3, BIRC2, TNFRSF1A associated via death domain (TRADD), RIPK1 and IKBKG] from the genes enriched in TNF, NF- $\mathrm{B}$ and apoptosis signaling pathways using Venn analysis (Fig. S1). A total of 12 overlapping genes were utilized to cross-check their genomic changes and clinical profiles in cBioPortal studies. After querying the overlapping gene set of all listed cancer studies, genomic alterations (top 10 cancer studies) were determined to range between 30.09 and $44.8 \%$ (Fig. 3A). As the study by Beltran et al (24) demonstrated the most prominent 
Neighboring genes connected to Glycyrrhizic acid-associated genes as filtered by alterations (\%)

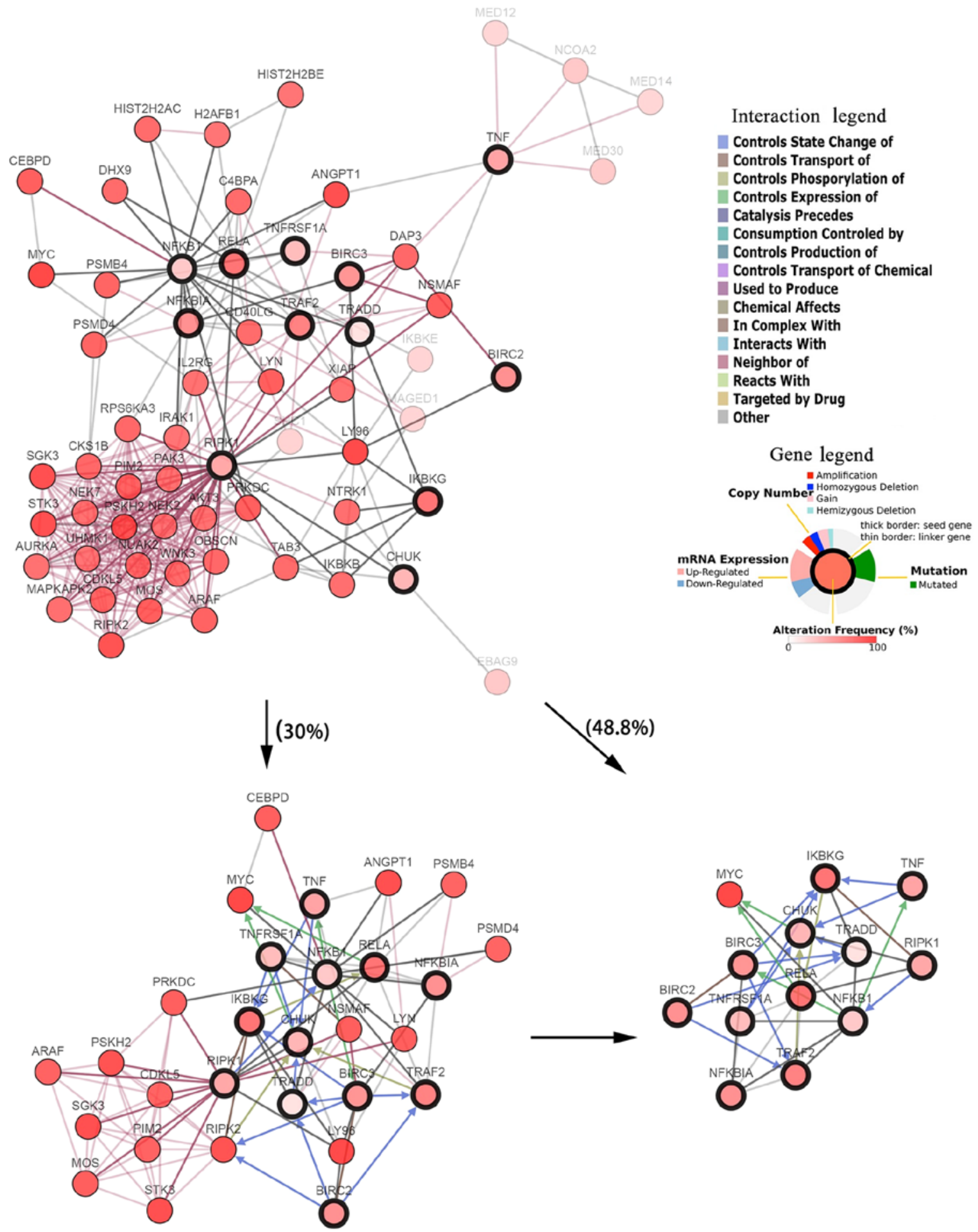

Figure 4. Visualized gene networks of GA-associated genes in neuroendocrine prostate cancer. Mined genes (TRAF2, TNF, NFKBIA, NFKB1, TNFRSF1A, CHUK, RELA, BIRC3, BIRC2, TRADD, RIPK1 and IKBKG; marked with a thick black border) were applied as seeds to capture their interactions, which were sequenced as altered genes in the current study. Neighbors are presented by adjusting filters to $30 \%$ and maximal genetic alterations.

genetic alterations, their queried genes were further explored in the current study. A visual summary of the documented genetic changes was created using OncoPrint and is presented in Fig. 3B. The results revealed that $48 \%$ of sequenced samples possessed an alteration in at least one gene, and that frequency changes ranged between 5 and $26 \%$, which were associated with the majority of gene amplifications. The query contained no genetic pairs with mutually exclusive alterations, and 66 gene pairs with concurrent alterations were determined, in which 27 gene pairs were significant $(\mathrm{P}<0.05$; data not shown).

Furthermore, cBioPortal provided interaction analyses for overlapping genes and built networks to display genes that were altered in neuroendocrine prostate cancer (Fig. 4). The current study constructed a network that comprised all 
neighbors of the overlapping genes from the pathway enrichment analysis. The network was then further modified by highlighting the neighbors of five key hub genes obtained from the PPI analysis. Five hub genes were also identified in crossed GA-associated secondary protein targets, including NFKB1, BIRC3, BIRC2, RIPK1 and IKBKG. To reduce the network complexity, only neighbors with higher alterations and queried genes were presented by filtering the frequency of genetic alterations. A gene network including the 12 overlapping genes and avian myelocytomatosis viral oncogene homolog (MYC; Entrez ID, 4609) was identified by filtering neighbor alterations ( $\geq 48.8 \%$ ). Comparatively, with the filter condition of neighbor alterations $\geq 30 \%$, a cluster of 29 genes were observed The complexity of interactions between the 12 GA-associated overlapping genes and their most relevant altered gene pairs in samples from the studies by Beltran et al (24) were presented on refined and unrefined networks.

\section{Discussion}

Licorice root is a traditional medicine that has been utilized to treat viral infections, peptic ulcers, lipidemia and hyperglycemia $(2,25)$. A considerable number of components have been extracted from licorice, including flavonoids and triterpenes, but GA is currently considered to be the main bioactive herbal compound (25). A wide range of pharmacological properties and multiple targets have been identified for GA via independent clinical and experimental studies. However, it is not fully known how GA serves a role in the regulation of certain biological processes. Therefore, a systematic method to bridge GA to its targets is required to connect these observed biological activities. The current study performed a PG-based analysis to elucidate the network interactions of GA and to assess its potential clinical application. PG-based analysis is comprised of four steps: i) GA is used as a seed to search for its primary direct targets by querying multiple drug bioinformatics and cheminformatics databases; ii) an assessment of the secondary indirect targets connected to GA-associated primary targets is performed by integrating data into a PPI network analysis tool; iii) pristine interaction networks associated with GA are filtered via GO and pathway enrichment analyses; and iv) the existence of genetic changes are validated for the refined target gene from large-scale samples obtained via cBioPortal cancer genomic studies. Different from experimental techniques that identify GA-associated targets, PG-based analysis is performed only via a rationalized hypothesis that integrates multiple online sources. PG-based analysis can generate effective results that may lead to the optimization of experiments to assess the complex mechanisms used by drugs.

The current study identified 5 GA-associated primary protein targets (HSD11B1, TNF, CASP3, NFKB2 and LPL), 138 functional partners of the primary targets, 30 enriched GO terms and 10 enriched pathways connected to GA-associated target genes. A target network center surrounding GA was then constructed based on the aforementioned results. Two obvious modules were determined in this network. Module one contained three primary targets (TNF, CASP3 and NFKB2) and 10 crossed secondary protein targets (BIRC3, BIRC2, FADD, PSMD2, IKBKG, UBC, RIPK1, NFKB1, PSMC3 and IL1B) that were closely associated with
TNF axis-mediated regulation of apoptosis, immunity and inflammation. TNF- $\alpha$ may therefore activate pro-apoptotic and anti-apoptotic pathways. TNF- $\alpha$ can induce apoptosis by activating caspase-family proteins (caspase-3, caspase- 8 and caspase-10). However, it may also inhibit apoptosis via $\mathrm{NF}-\kappa \mathrm{B}$, which induces the expression of anti-apoptotic genes including Bcl-2 $(22,26)$. In addition, cytokines of the TNF family may induce gene transcription to regulate inflammation, cell survival, cell differentiation and cell proliferation, primarily via the activation of the $\mathrm{NF}-\kappa \mathrm{B}$ pathway (27). Module two contained two primary targets (HSD11B1 and LPL) and two crossed secondary protein targets (EHHADH and $\mathrm{NR} 3 \mathrm{C} 1$ ) that were closely associated with the regulation of lipometabolism (28). Functional characteristic analysis of 129 GA-associated target genes was performed via KEGG. It was revealed that 34 genes were classified as validated targets of GA and were significantly enriched in the TNF signaling pathway. BP analysis of GA-associated target genes revealed that $29.5 \%$ of genes were enriched in 'TNF-mediated signaling pathway', which was the most statistically significant response. Module 1, associated with the TNF axis, was then considered to be the core analysis subset, and it was speculated that bioactivities may be initiated via the interaction between GA and its targets at the origin of various molecular events.

The TNF pathway is strongly associated with the physiological or pathological regulation of apoptosis, cell survival, cell proliferation and inflammation (22). In recent years, the GA-mediated regulation of inflammation has been studied, particularly in hepatoprotective processes. However the GA-mediated regulation of apoptosis, cell proliferation and cell survival in certain diseases, such as cancer, remains unclear. Connectivity between the TNF axis and GA-mediated biological functions in cancer were assessed by determining the genetic changes of 12 overlapping genes (TRAF2, TNF, NFKBIA, NFKB1, TNFRSF1A, CHUK, RELA, BIRC3, BIRC2, TRADD, RIPK1 and IKBKG) revealed by TNF and TNF-associated signaling pathways $(\mathrm{TNF} / \mathrm{NF}-\kappa \mathrm{B}$ signaling pathway and $\mathrm{TNF} / \mathrm{caspase}$ family apoptosis signaling pathway). In the current study, the connectivity between GA-associated targets and cancer was revealed via queries made using cBioPortal. In the case of neuroendocrine prostate cancer, frequency changes of the gene set ranged between 5 and $26 \%$, and the abrupt genetic alterations centered on expression amplification. RELA (with a $26 \%$ change) is a proto-oncogene that usually forms a complex with NFKB, which subsequently moves to the nucleus and activates the transcription of specific genes (29). IKBKG (26\%), an NF- $\kappa \mathrm{B}$ essential modulator, encodes the subunit of the inhibitor of $\mathrm{NF}-\kappa \mathrm{B}$ kinase complex, which activates $\mathrm{NF}-\kappa \mathrm{B}$ and induces the activation of genes relating to survival, inflammation and other pathways. Similarly to RELA, hypomorphic mutations of IKBKG may result in immunodeficiency (30). TRAF2 (22\%), a mediator of TNF receptor anti-apoptotic signaling, is indispensable for the TNF- $\alpha$-mediated activation of MAPK8/JNK and NF- $\kappa B$ (31). TRADD, a TNF receptor-associated apoptotic signal transducer, interacts with TRAF2, resulting in the direct inhibition of caspase activation by recruiting inhibitor-of-apoptosis proteins (IAPs) (32). In addition, other IAPs inhibit apoptosis. For example, BIRC2 (19\%) and BIRC3 (18\%) usually form a complex by binding to TRAF1/2 $(33,34)$. Previous studies 
have indicated that the anti-cancer activities of GA are closely associated with anti-proliferative or anti-angiogenic signals linked to the TNF axis $(5,9,35,36)$. Therefore, the results of the PG-based analysis indicated that GA-associated core targets may therapeutically benefit neuroendocrine prostate cancer.

It has been established that TNF binds to TNFR $1 / 2$ to regulate distinct downstream pathways under certain conditions. The binding of TNF to TNFR1 activates MAPK/NF- $\kappa \mathrm{B} /$ caspase signaling pathways to mediate the regulation of inflammatory cytokines, vascular effects or apoptosis (37). In addition, binding may activate the PI3K-AKT signaling pathway to mediate the regulation of cell survival (37). Three primary targets (TNF, CASP3 and NFKB2) of the TNF axis exhibit GA-mediated inhibitory activity. TNFR1 of the $\mathrm{TNF} / \mathrm{NF}-\kappa \mathrm{B}$ signaling pathway and TNFR2 of the PI3K-AKT signaling pathway are considered to be the most relevant targets. However, the PI3K-AKT signaling pathway was not included in the top 10 pathways of KEGG. The GA-mediated downregulation of this pathway has been previously demonstrated as an important mechanism involved in the anti-cancer effects of GA in the growth and migration of leukemia cells (5). Therefore, the current study may only provide a reference for future biological studies. Despite this, significant genetic alterations of TRAF2 (22\%), BIRC2 19\%) and BIRC3 (18\%) were identified in the current study and may therefore serve primary regulatory roles.

The PG-based analysis of the present study was further utilized to mine potential interactions connected to GA-associated overlapping targets in neuroendocrine prostate cancer by constructing a visual network using cBioPortal. It is possible to identify key targets for the treatment of neuroendocrine prostate cancer. Although neuroendocrine prostate cancer is extremely rare, with a prevalence of $<1 \%$, it is a lethal and aggressive subtype of prostate cancer, which includes the resistance mechanism of non-androgen receptors (38). The loss or inactivation of RB transcriptional corepressor 1 and p53, or the overexpression of the MYC family (particularly $\mathrm{N}-\mathrm{My}$ and $\mathrm{C}-\mathrm{Myc}$ ) serve important roles in the development of aggressive prostate cancer and metastasis (38). The current study revealed that the MYC target gene (44.8\%) exhibited the highest alteration by filtering the interactive network connected to overlapping targets associated with GA. Furthermore, RIPK1 (14\%) was deemed to serve an important role as it connected 12 of the 29 genes with $>30 \%$ genetic alterations in neuroendocrine prostate cancer. The majority of targets that interacted with RIPK1 were considered to be proto-oncogenes, including LYN proto-oncogene, Src family tyrosine kinase (33.6\%), A-Raf proto-oncogene, serine/threonine kinase $(30.8 \%)$, Pim-2 proto-oncogene, serine/threonine kinase $(31.8 \%)$ and MOS proto-oncogene, serine/threonine kinase $(33.6 \%)$. In response to tissue damage and pathogen recognition, the encoded protein of RIPK1 serves an important role in the regulation of inflammation and cell death (39). This may provide an increased understanding of underlying malignant disease mechanisms and may lead to the development of GA-associated chemoprevention in neuroendocrine prostate cancer.

However, certain previously demonstrated effects of GA were not identified among candidate genes in the present study. For example, high mobility group box 1 (HMGB1) is a nuclear DNA-binding protein that regulates transcription in several cellular processes including inflammation, cell differentiation and tumor cell migration (40). In particular, the impact of GA on HMGB1 is of great biological relevance to the pharmacological effects of GA in immunoinflammatory diseases. GA has been demonstrated to inhibit HMGB1 expression, resulting in the upregulation of heme oxygenase 1 expression (41). By inhibiting the interaction of HMGB1 with its receptor toll like receptor 2, GA may have the potential to inhibit the activation of the innate immune system, including the production of IL-1 and TNF- $\alpha$, which are implicated in the pathogenesis of immunoinflammatory and autoimmune diseases such as multiple sclerosis and rheumatoid arthritis (42). Although biological evidence indicates that the GA-mediated inhibition of HMGB1 is a principal pharmacological effect, the PG analysis of the current study did not determine HMGB1 as target of GA. The reason why certain demonstrated targets in vitro, such as HMGB1 and the inflammasome, were not shown in the present analysis may that the interaction is indirect, and thus these are not primary targets. Alternatively, it may be that the databases were not up-to-date with respect to predicting the secondary interactions. For this reason, it is hypothesized that other genes not included in the analysis of the current study remain worthy of assessment in vitro to determine all the pharmacological effects of GA; in particular, the effects of GA on the pleiotropic cytokine macrophage migration inhibitory factor, which is implicated in the pathogenesis of autoimmune diseases and cancer, and is an activator of the inflammasome (43). There remain many obstacles to validating associations based on PG analysis and their translation into clinical practice. Although $\sim 15 \%$ of approved drugs include PG information on the label, only a fraction of these are deemed actionable (44).

Based on the PG analysis strategy implemented in the current study, a network of 138 functional interactions was constructed to identify GA-associated primary and secondary targets. The main biological effects mediated by the drug-target network of GA were enriched via the regulation of the TNF axis, as determined using integrated analyses. The impacts of TNF axis core elements were further evaluated in a specific type of cancer via the cBioPortal altered genes network. A simple and flexible interface was also constructed to test hypotheses regarding significant genetic alterations and subsequent phenotype expression, and to develop the potential use of GA in neuroendocrine prostate cancer. Furthermore, the analytical method used in the current study may help elucidate the side effects of GA. However, further experimentation is required to determine which effects are dominant in the treatment of neuroendocrine prostate cancer, therapeutic or side effects.

\section{Acknowledgements}

The authors would like to thank Miss Caina Gao (Affiliated Children's Hospital of Zhejiang University), Miss Jie Bao and Dr Hang Mu (both (Yangtze Delta Region Institute of Tsinghua University) for assisting in the preparation of the manuscript.

\section{Funding}

No funding was received. 


\section{Availability of data and materials}

The datasets used and/or analyzed during the current study are available from the corresponding author on reasonable request.

\section{Authors' contributions}

SW, LL and LS conceived and designed the current study. SW and LL performed data analysis. SW wrote the manuscript. SW and LL revised the manuscript. All authors read and approved the final manuscript.

\section{Ethics approval and consent to participate}

Not applicable.

\section{Patient consent for publication}

Not applicable.

\section{Competing interests}

The authors declare that they have no competing interests.

\section{References}

1. Saif MW, Li J, Lamb L, Kaley K, Elligers K, Jiang Z, Bussom S, Liu SH and Cheng YC: First-in-human phase II trial of the botanical formulation PHY906 with capecitabine as second-line therapy in patients with advanced pancreatic cancer. Cancer Chemother Pharmacol 73: 373-380, 2014.

2. Ming LJ and Yin AC: Therapeutic effects of glycyrrhizic acid. Nat Prod Commun 8: 415-418, 2013.

3. Farrukh MR, Nissar UA, Kaiser PJ, Afnan Q, Sharma PR, Bhushan S and Tasduq SA: Glycyrrhizic acid (GA) inhibits reactive oxygen Species mediated photodamage by blocking ER stress and MAPK pathway in UV-B irradiated human skin fibroblasts. J Photochem Photobiol B 148: 351-357, 2015.

4. Fouladi S, Masjedi M, Ghasemi R, G Hakemi M and Eskandari N: The in vitro impact of glycyrrhizic acid on $\mathrm{CD}^{+} \mathrm{T}$ lymphocytes through OX40 receptor in the patients with allergic rhinitis. Inflammation 41: 1690-1701, 2018.

5. He SQ, Gao M, Fu YF and Zhang YN: Glycyrrhizic acid inhibits leukemia cell growth and migration via blocking AKT/mTOR/STAT3 signaling. Int J Clin Exp Pathol 8: 5175-5181, 2015.

6. Hou S, Zhang T, Li Y, Guo F and Jin X: Glycyrrhizic acid prevents diabetic nephropathy by activating AMPK/SIRT1/PGC-1 $\alpha$ signaling in db/db mice. J Diabetes Res 2017: 2865912, 2017.

7. Kiratipaiboon $\mathrm{C}$, Tengamnuay $\mathrm{P}$ and Chanvorachote $\mathrm{P}$ : Glycyrrhizic acid attenuates stem cell-like phenotypes of human dermal papilla cells. Phytomedicine 22: 1269-1278, 2015.

8. Li C, Peng S, Liu X, Han C, Wang X, Jin T, Liu S, Wang W, Xie X, He X, et al: Glycyrrhizin, a direct HMGB1 antagonist, ameliorates inflammatory infiltration in a model of autoimmune thyroiditis via inhibition of TLR2-HMGB1 signaling. Thyroid 27: 722-731, 2017.

9. Lin SC, Chu PY, Liao WT, Wu MY, Tsui KH, Lin LT, Huang CH, Chen LL and Li CJ: Glycyrrhizic acid induces human MDA-MB-231 breast cancer cell death and autophagy via the ROS-mitochondrial pathway. Oncol Rep 39: 703-710, 2018.

10. Refahi S, Pourissa M, Zirak MR and Hadadi G: Modulation expression of tumor necrosis factor $\alpha$ in the radiation-induced lung injury by glycyrrhizic acid. J Med Phys 40: 95-101, 2015.

11. Wang LY, Cheng KC, Li Y, Niu CS, Cheng JT and Niu HS: Glycyrrhizic acid increases glucagon like peptide-1 secretion via TGR5 activation in type 1-like diabetic rats. Biomed Pharmacother 95: 599-604, 2017.

12. Wang YM and Du GQ: Glycyrrhizic acid prevents enteritis through reduction of NF- $\kappa \mathrm{B}$ p 65 and p38MAPK expression in rat. Mol Med Rep 13: 3639-3646, 2016.
13. Yang J, Shi Y, Chen H, Wang X, Chen Y and Yang B: Glycyrrhizic acid attenuates myocardial injury: Involvement of RIP140/NF- $\mathrm{B}$ Pathway. Biomed Pharmacother 95: 62-67, 2017.

14. Zhang H, Zhang R, Chen J, Shi M, Li W and Zhang X: High mobility group Box 1 inhibitor glycyrrhizic acid attenuates kidney injury in streptozotocin-induced diabetic rats. Kidney Blood Press Res 42: 894-904, 2017.

15. Relling MV and Evans WE: Pharmacogenomics in the clinic. Nature 526: 343-350, 2015.

16. Mooney SD: Progress towards the integration of pharmacogenomics in practice. Hum Genet 134: 459-465, 2015.

17. Campedel L, Kossaï M, Blanc-Durand P, Rouprêt M, Seisen T, Compérat E, Spano JP and Malouf G: Neuroendocrine prostate cancer: Natural history, molecular features, therapeutic management and future directions. Bull Cancer 104: 789-799, 2017 (In French).

18. Su G, Morris JH, Demchak B and Bader GD: Biological network exploration with Cytoscape 3. Curr Protoc Bioinformatics 47: 8.13.1-24, 2014.

19. Guo Y, Bao Y, Ma M and Yang W: Identification of key candidate genes and pathways in colorectal cancer by integrated bioinformatical analysis. Int J Mol Sci 18: pii: E722, 2017.

20. Gao J, Aksoy BA, Dogrusoz U, Dresdner G, Gross B, Sumer SO, Sun Y, Jacobsen A, Sinha R, Larsson E, et al: Integrative analysis of complex cancer genomics and clinical profiles using the cBioPortal. Sci Signal 6: pl1, 2013.

21. Canault B, Bourg S, Vayer P and Bonnet P: Comprehensive network map of ADME-Tox databases. Mol Inform 36, 2017.

22. Coelho-Santos V, Leitão RA, Cardoso FL, Palmela I, Rito M, Barbosa M, Brito MA, Fontes-Ribeiro CA and Silva AP: The TNF- $\alpha / N F-\kappa B$ signaling pathway has a key role in methamphetamine-induced blood-brain barrier dysfunction. J Cereb Blood Flow Metab 35: 1260-1271, 2015.

23. Kang R, Xie Y, Zhang Q, Hou W, Jiang Q, Zhu S, Liu J, Zeng D, Wang H, Bartlett DL, et al: Intracellular HMGB1 as a novel tumor suppressor of pancreatic cancer. Cell Res 27: 916-932, 2017.

24. Beltran H, Prandi D, Mosquera JM, Benelli M, Puca L, Cyrta J, Marotz C, Giannopoulou E, Chakravarthi BV, Varambally S, et al: Divergent clonal evolution of castration-resistant neuroendocrine prostate cancer. Nat Med 22: 298-305, 2016.

25. Asl MN and Hosseinzadeh H: Review of pharmacological effects of Glycyrrhiza sp. and its bioactive compounds. Phytother Res 22: 709-724, 2008.

26. Brumatti G, Salmanidis M and Ekert PG: Crossing paths: Interactions between the cell death machinery and growth factor survival signals. Cell Mol Life Sci 67: 1619-1630, 2010.

27. Hayden MS and Ghosh S: Regulation of NF- $\kappa$ B by TNF family cytokines. Semin Immunol 26: 253-266, 2014.

28. Yaw HP, Ton SH, Chin HF, Karim MK, Fernando HA and Kadir KA: Modulation of lipid metabolism in glycyrrhizic acid-treated rats fed on a high-calorie diet and exposed to short or long-term stress. Int J Physiol Pathophysiol Pharmacol 7: 61-75, 2015.

29. Chen S, Jiang S, Zheng W, Tu B, Liu S, Ruan H and Fan C: RelA/p65 inhibition prevents tendon adhesion by modulating inflammation, cell proliferation, and apoptosis. Cell Death Dis 8: e2710, 2017.

30. Miot C, Imai K, Imai C, Mancini AJ, Kucuk ZY, Kawai T, Nishikomori R, Ito E, Pellier I, Dupuis Girod S, et al: Hematopoietic stem cell transplantation in 29 patients hemizygous for hypomorphic IKBKG/NEMO mutations. Blood 130: 1456-1467, 2017.

31. Wei B, Liang J, Hu J, Mi Y, Ruan J, Zhang J, Wang Z, Hu Q, Jiang $\mathrm{H}$ and Ding Q: TRAF2 is a valuable prognostic biomarker in patients with prostate cancer. Med Sci Monit 23: 4192-4204, 2017.

32. Wei B, Ruan J, Mi Y, Hu J, Zhang J, Wang Z, Hu Q, Jiang H and Ding Q: Knockdown of TNF receptor-associated factor 2 (TRAF2) modulates in vitro growth of TRAIL-treated prostate cancer cells. Biomed Pharmacother 93: 462-469, 2017.

33. Jin H, Dong YY, Zhang H, Cui Y, Xie K and Lou G: shRNA depletion of cIAP1 sensitizes human ovarian cancer cells to anticancer agent-induced apoptosis. Oncol Res 22: 167-176, 2014.

34. Seidelin JB: Regulation of antiapoptotic and cytoprotective pathways in colonic epithelial cells in ulcerative colitis. Scand J Gastroenterol 50 (Suppl 1): S1-S29, 2015.

35. Hostetler BJ, Uchakina ON, Ban H and McKallip RJ: Treatment of hematological malignancies with glycyrrhizic acid. Anticancer Res 37: 997-1004, 2017. 
36. Kim KJ, Choi JS, Kim KW and Jeong JW: The anti-angiogenic activities of glycyrrhizic acid in tumor progression. Phytother Res 27: 841-846, 2013.

37. Doss GP, Agoramoorthy G and Chakraborty C: TNF/TNFR: Drug target for autoimmune diseases and immune-mediated inflammatory diseases. Front Biosci (Landmark Ed) 19: 1028-1040, 2014

38. Berman-Booty LD and Knudsen KE: Models of neuroendocrine prostate cancer. Endocr Relat Cancer 22: R33-R49, 2015.

39. Guo R, Tu Y, Xie S, Liu XS, Song Y, Wang S, Chen X and Lu L: A role for receptor-interacting protein kinase-1 in neutrophil extracellular trap formation in patients with systemic lupus erythematosus: A preliminary study. Cell Physiol Biochem 45: 2317-2328, 2018.

40. Ando K, Sakoda M, Ueno S, Hiwatashi K, Iino S, Minami K, Kawasaki Y, Hashiguchi M, Tanoue K and Mataki Y, et al: Clinical implication of the relationship between high mobility group Box-1 and tumor differentiation in hepatocellular carcinoma. Anticancer Res 38: 3411-3418, 2018.

41. Zhang Y, Li L, Qi C, Hua S, Fei X, Gong F and Fang M: Glycyrrhizin alleviates Con A-induced hepatitis by differentially regulating the production of IL-17 and IL-25. Biomed Pharmacother 110: 692-699, 2019.
42. Dujmovic I, Mangano K,Pekmezovic T, Quattrocchi C, Mesaros S, Stojsavljevic N, Nicoletti F and Drulovic J: The analysis of IL-1 beta and its naturally occurring inhibitors in multiple sclerosis: The elevation of IL-1 receptor antagonist and IL-1 receptor type II after steroid therapy. J Neuroimmunol 207: 101-106, 2009.

43. Shin MS, Kang Y, Wahl ER, Park HJ, Lazova R, Leng L, Mamula M, Krishnaswamy S, Bucala R and Kang I: Macrophage migration inhibitory factor regulates U1 small nuclear RNP immune complex-mediated activation of the NLRP3 inflammasome. Arthritis Rheumatol 71: 109-120, 2019.

44. Ehmann F, Caneva L, Prasad K, Paulmichl M, Maliepaard M, Llerena A, Ingelman-Sundberg $M$ and Papaluca-Amati M: Pharmacogenomic information in drug labels: European medicines agency perspective. Pharmacogenomics J 15: 201-210, 2015.

This work is licensed under a Creative Commons Attribution-NonCommercial-NoDerivatives 4.0 International (CC BY-NC-ND 4.0) License. 